\title{
Efficacy and Safety of Insulin Glulisine in Intensive Insulin Therapy: Bolus Insulin Adjust Nice Control by apiDRA Study (BANDRA Study)
}

\author{
Yukihiro Bando*, Kousuke Shima, Keiko Aoki, Hideo Kanehara, Azusa Hisada, \\ Kazuhiro Okafuji, Daisyu Toya, Nobuyoshi Tanaka \\ Department of Internal Medicine, Fukui-ken Saiseikai Hospital, Fukui, Japan \\ Email: ${ }^{*} y$-bando@fukui.saiseikai.or.jp, shimaccyo@gmail.com, k-aoki@fukui.saiseikai.or.jp, \\ h-kanehara@fukui.saiseikai.or.jp, a-hisada @fukui.saiseikai.or.jp, k-okafuji@fukui.saiseikai.or.jp, \\ d-toya@fukui.saiseikai.or.jp, n-tanaka@fukui.saiseikai.or.jp
}

Received 18 December 2014; accepted 2 January 2015; published 15 January 2015

Academic Editor: Sharma S. Prabhakar, Texas Tech University Health Sciences Center, USA

Copyright (C) 2015 by authors and Scientific Research Publishing Inc.

This work is licensed under the Creative Commons Attribution International License (CC BY). http://creativecommons.org/licenses/by/4.0/

(c) (i) Open Access

\section{Abstract}

Background: Treatment for postprandial glycemia using rapid-acting insulin analogues sometimes resulted in preprandial hypoglycemia or weight gain. Objective: This study evaluated the efficacy and safety of switching bolus insulin from insulin lispro (Lis) to insulin glulisine (Glu) in patients with inadequately controlled diabetes on intensive insulin therapy with Lis and glargine (Gla). Methods: Seventy-two outpatients with inadequate glycemic control (glycated hemoglobin [HbA1c] $\geq 7.0 \%$, glycated albumin $[\mathrm{GA}] \geq \mathbf{2 0 \%}$ ) on intensive insulin therapy comprising Lis and Gla for $\geq 24$ weeks were enrolled. We switched treatment from Lis to Glu with a stepwise increase in the dose by 1 unit per meal to obtain a GA level of $\leq 20 \%$ for 24 weeks, and the efficacy and safety were evaluated. Patients' treatment satisfaction was also evaluated using the Diabetes Treatment Satisfaction Questionnaire (DTSQ) after the treatment. Results: After switching from Lis to Glu, both HbA1c and GA levels significantly lowered from $8.26 \% \pm 0.13 \%$ to $7.71 \% \pm 0.13 \%(P<0.01)$ and from $23.9 \% \pm 1.8 \%$ to $21.4 \% \pm 1.9 \%(P<0.01)$, respectively. Furthermore, switching from Lis to Glu improved patients' treatment satisfaction; scores for 7 of the 8 items, such as "satisfaction" and "convenience" were significantly improved $(P<0.001)$, with no significant change in the scores for "improvement of hypoglycemia" $(P=0.91)$. Conclusions: Our present study suggests that switching bolus insulin from Lis to Glu by the addition of 1 unit of Glu per meal may be a use-

${ }^{*}$ Corresponding author.

How to cite this paper: Bando, Y., Shima, K., Aoki, K., Kanehara, H., Hisada, A., Okafuji, K., Toya, D. and Tanaka, N. (2015) Efficacy and Safety of Insulin Glulisine in Intensive Insulin Therapy: Bolus Insulin Adjust Nice Control by apiDRA Study (BANDRA Study). Journal of Diabetes Mellitus, 5, 28-35. http://dx.doi.org/10.4236/jdm.2015.51004 
ful treatment option for patients with inadequate glycemic control receiving intensive insulin therapy with Lis and Gla.

\author{
Keywords
}

\author{
Insulin Glulisine, Insulin Lispro, Intensive Insulin Therapy
}

\title{
1. Introduction
}

The purpose of diabetes management is improvement of quality of life (QOL) and healthy life expectancy by preventing the development or progression of blood vessel complications. Therefore, diabetes management is important for the management of macroangiopathy as well as microangiopathy including retinopathy, nephropathy, and neuropathy. Many studies have already reported a greater risk of coronary heart disease (CHD), including myocardial infarction and angina, in patients with diabetes than in individuals without diabetes [1] [2]. In particular, as an indicator of the risk of cardiovascular events, the 2-h postload glucose level on oral glucose tolerance test (OGTT) has been reported as important in the DECODE Study (Diabetes Epidemiology: Collaborative Analysis of Diagnostic Criteria in Europe) [3], which is a meta-analysis of 13 cohort studies in the EU, and the Funagata Study [4] conducted in Funagata-cho in Japan. In contrast, in the Honolulu Heart Study [5], which included 6394 Japanese individuals living in Hawaii with no history of CHD or stroke, OGTT was performed in all cases and the 1-hour postload glucose level on OGTT results were analyzed. Results were that in the highest group of 1-h postprandial glycemia, death risk was approximately 3 times higher than the lowest group.

In this context, the International Diabetes Federation established the Guideline for Management of PostMeal Glucose that was the first guideline to emphasize the importance of the 2-h OGTT in 2007. The revised edition of the Guideline for Management of PostMeal Glucose in 2011 [6] proposed to conduct an examination of postprandial glycemia within 1 - 2 hours in order to inhibit blood vessel complications, to reduce it to less than 160 $\mathrm{mg} / \mathrm{dL}(9.0 \mathrm{mmol} / \mathrm{L})$ avoiding hypoglycemia. However, treatment for postprandial glycemia using rapid-acting insulin analogues such as insulin lispro (Lis) or insulin aspart (Asp) resulted in preprandial hypoglycemia or weight gain; therefore, an appropriate dose of bolus insulin could not be administered [7] [8].

On the other hand, insulin glulisine (Glu) exists largely as a monomer in products; in this form, it mimics the secretion of insulin more physiologically than Lis and Asp, as it is rapidly absorbed and immediately excreted in the bloodstream after subcutaneous injection (application document of Sanofi-Aventis: currently Sanofi).

In the Japanese clinical trial [8], it was reported that frequency of hypoglycemia decreased after switching from Lis to Glu at the same dose, and did not increase by the stepwise increase in Glu dose by 1 unit that was added to every meal. These results suggest that, by switching from Lis to Glu with 1-unit increments allows adequate bolus insulin provision to patients with inadequate postprandial glycemic control with Lis, without increasing the risk of hypoglycemia.

In this preliminary design of study, we aimed to assess the efficacy and safety of bolus insulin that was switched from Lis to Glu with an additional dose by of 1 unit per meal in patients receiving intensive insulin therapy with Lis and Gla.

\section{Methods}

\subsection{Study Population}

Subjects included 72 outpatients with type $1(n=21)$, type $2(n=48)$, and other type $(n=3)$ diabetes and inadequate glycemic control (glycated hemoglobin $[\mathrm{HbA1c}] \geq 7.0 \%$ and glycated albumin $[\mathrm{GA}] \geq 20 \%$ ) despite intensive insulin therapy with Lis and Gla as bolus insulin therapy for $>24$ weeks under a fixed titration protocol; the dose of Lis and Gla was titrated in a stepwise manner to achieve a GA level of $\leq 20 \%$ on the discretion of the treating physician. Written informed consent was obtained from all patients.

The exclusion criteria were as follows: 1) need for hypoglycemic drug therapy besides Glu and Gla during the observation period; 2) history of hypersensitivity to Glu; 3) history of severe ketoacidosis or diabetic coma or precoma; 4) severe infectious disease or severe trauma after surgery; 5) pregnancy or suspected pregnancy; 6) 
medical or family history of hypothyroidism and hereditary muscular disorders (e.g. muscular dystrophy); 7) history of drug-induced hepatic disorders; 8) drug or alcohol addiction; or 9) contraindications identified by the treating physician.

\subsection{Study Design}

This study was a single center, prospective, open-label, before-after study conducted from 1 April 2011 to 31 November 2013. We switched treatment from Lis to Glu with by the addition of 1 unit per meal at the time of presentation. Thereafter, the dose of Glu was increased in a stepwise manner to achieve a GA level of $\leq 20 \%$ on the discretion of the treating physician using the same titration protocol used for Lis. To compare the effects of Glu with those of Lis selectively, the dose of Gla was not changed for 12 weeks after switching. After improvement of HbA1c or fasting plasma glucose level was judged to be insufficient after 12 weeks, the dose of Gla was increased and levels were monitored for 24 weeks. After the insulin Gla plus Glu combination therapy, we evaluated patients' treatment satisfaction by using the Diabetes Treatment Satisfaction Questionnaire (DTSQ) [9] that included 8 items ("satisfaction", "improvement of hyperglycemia", "improvement of hypoglycemia", "convenience", "versatility", "recommendable to other patients", "understanding”, and "treatment continuity") graded from -3 (mostly unsatisfied) to +3 (mostly satisfied), and compared the scores on the former treatment with insulin Gla plus Lis combination therapy. This study is registered with the University Hospital Medical Information Network Clinical Trials Registry (UMIN-CTR; Japan), number UMIN000008797.

\subsection{Endpoints}

The primary endpoints of this study were the change in HbA1c and GA levels after treatment, and the achievement of HbA1c level $\leq 7.0 \%$ and GA $\leq 20.0 \%$. Secondary endpoints were the change in the GA/HbA1c ratio, body weight, insulin dose, and degree of patients' treatment satisfaction as evaluated by the DTSQ.

\subsection{Statistical Analysis}

The values were expressed as mean \pm standard deviation (S.D.) or mean \pm standard error (S.E.). Differences between two variables were examined using the two-tailed paired Student's $t$ tests. $P<0.05$ was considered statistically significant. All statistical analyses were performed with StatView version 5.0 for Windows (SAS Institute, Cary, NC).

\section{Results}

\subsection{Patient Characteristics}

Patient characteristics are shown in Table 1 . Among the 72 patients with diabetes, 47 were men and 25 were women. Twenty-one patients had type 1 diabetes (including 1 with fulminant type 1 diabetes mellitus and 2 with

Table 1. Patient characteristics at baseline.

\begin{tabular}{cc}
\hline Characteristics & Patients ( $=72)$ \\
\hline Sex (male/female) & $47 / 25$ \\
Types of diabetes (type 1:type 2:other) & $21: 48: 03$ \\
Age (years) & $59.4 \pm 14.0$ \\
Diabetes duration (years) & $10.3 \pm 6.0$ \\
BMI (kg/m $\left.{ }^{2}\right)$ & $22.4 \pm 4.2$ \\
HbA1c (\%) & $8.26 \pm 1.07$ \\
GA (\%) & $23.9 \pm 6.1$ \\
Bolus insulin dose (IU/day) & $22.3 \pm 9.4$ \\
Basal insulin dose (IU/day) & $10.1 \pm 8.0$ \\
\hline
\end{tabular}


slowly progressive insulin-dependent diabetes mellitus), 48 had type 2 diabetes, and 3 had other types of diabetes ( 2 with post-pancreatectomy and 1 with steroid-induced diabetes). The mean age was $59.4 \pm 14.0$ years, mean duration of disease was $10.3 \pm 6.0$ years, mean body mass index (BMI) was $22.4 \pm 4.2 \mathrm{~kg} / \mathrm{m}^{2}$, mean HbA1c level was $8.26 \% \pm 1.07 \%$, and mean GA level was $23.9 \% \pm 6.1 \%$. Before switching, the mean unit of basal insulin (Gla) was $10.1 \pm 8.0$ unit/day, and the mean unit of bolus insulin (Lis) was $22.3 \pm 9.4$ unit/day.

\subsection{Changes in Clinical Parameters}

Changes in HbA1c and GA levels are shown in Figure 1 and Figure 2. The mean HbA1c level significantly decreased from $8.26 \% \pm 0.13 \%$ before the switch to $7.71 \% \pm 0.13 \%(P<0.01)$ after 24 weeks. Significant decrease in the HbA1c level was seen in both type 1 and type 2 diabetic patients with levels that decreased from

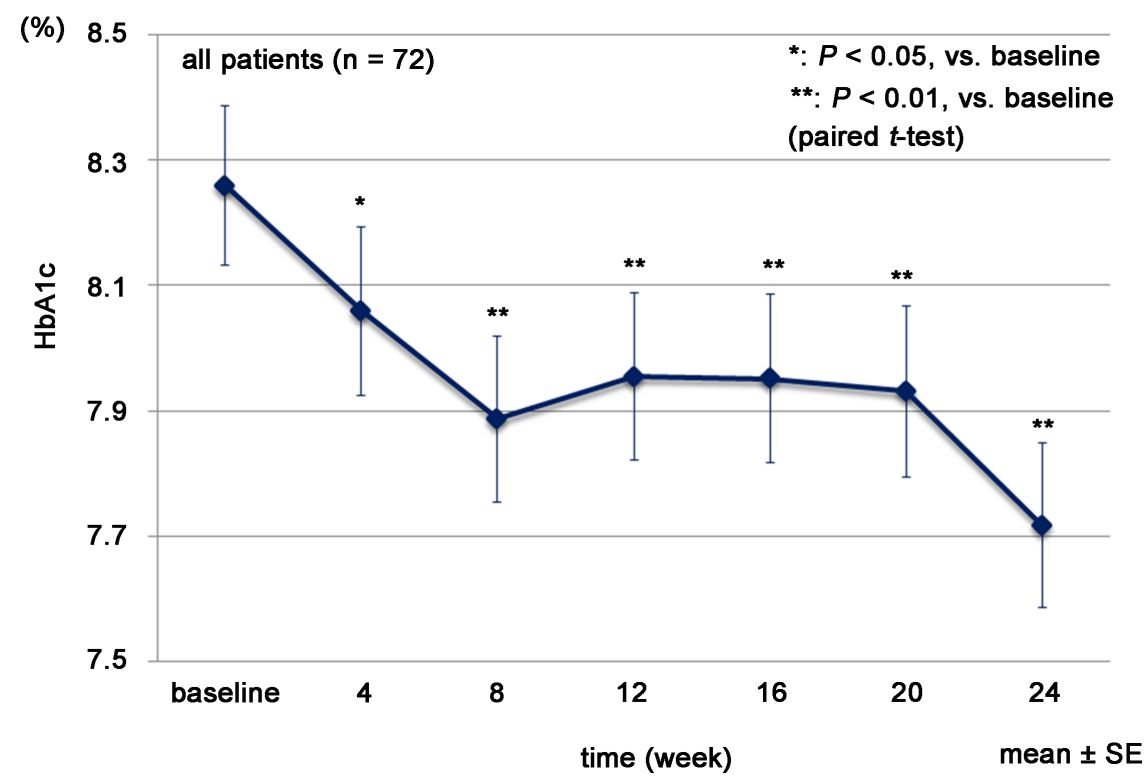

Figure 1. Changes in HbA1c from baseline after switching to insulin glulisine.

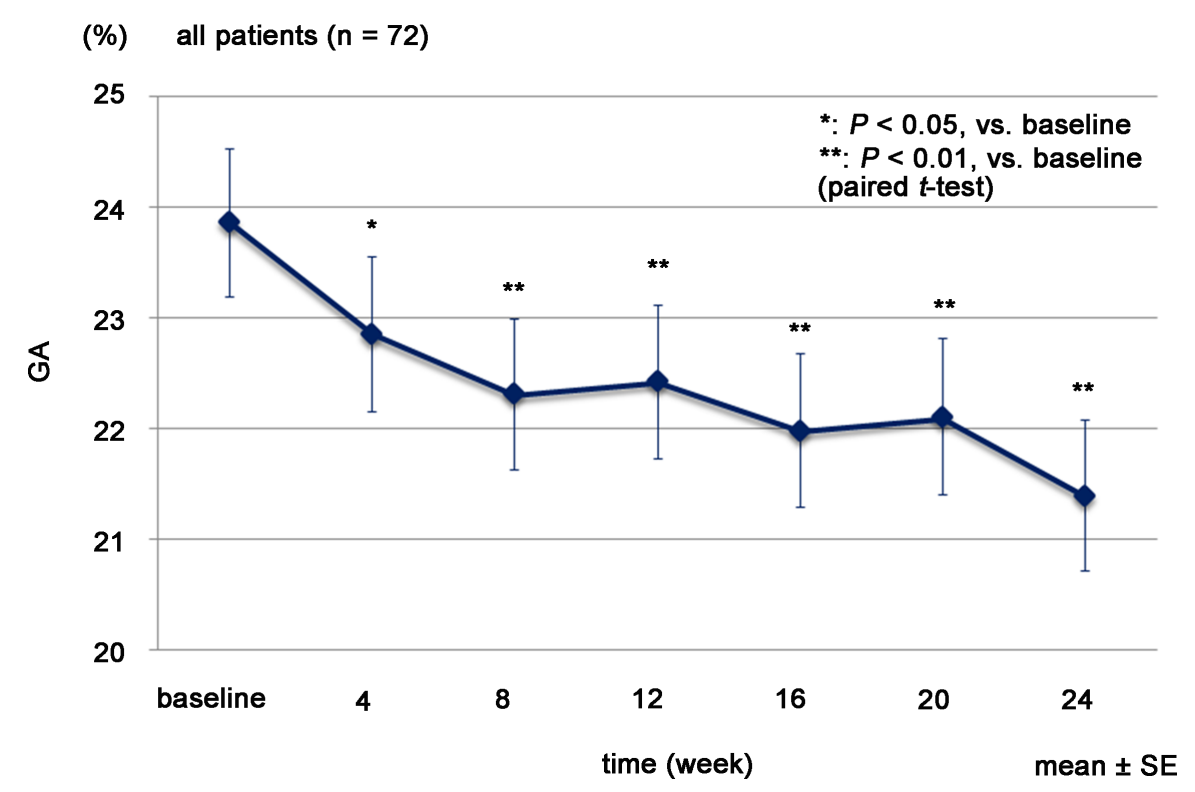

Figure 2. Changes in GA from baseline after switching to insulin glulisine. Abbreviations: GA, glycated albumin. 
$8.37 \% \pm 0.26 \%$ to $7.99 \% \pm 0.26 \%(P=0.004)$ and from $8.20 \% \pm 0.15 \%$ to $7.61 \% \pm 0.16 \%(P<0.001)$, respectively. Similarly, GA level significantly improved from $23.9 \% \pm 1.8 \%$ to $21.4 \% \pm 1.9 \%(P<0.01)$. The proportion of patients who achieved HbAlc levels $\leq 7.0 \%$ and GA levels $\leq 20.0 \%$ was $24.6 \%(19 / 72)$ and $41.7 \%$ (30/72), respectively.

Switching from Lis to Glu did not significantly improve the GA/HbA1c ratio after 24 weeks (from $2.80 \pm 0.6$ to $2.68 \pm 0.5, P=0.12$ ). Mean body weight increased significantly from $57.7 \pm 2.8 \mathrm{~kg}$ to $58.1 \pm 2.8 \mathrm{~kg}(P=0.02)$ in patients with type 1 diabetes, although there was no significant change in patients with type 2 diabetes (from $69.8 \pm 7.5 \mathrm{~kg}$ to $68.7 \pm 8.5 \mathrm{~kg}, P=0.91)$.

Changes in insulin dose are shown in Figure 3. In all patients, the bolus insulin dose significantly increased from $22.3 \pm 1.2$ unit/day of Lis before switching to $26.8 \pm 1.2$ unit/day of Glu after 24 weeks $(P<0.001)$. The dose of Gla as basal insulin increased significantly from $10.1 \pm 1.00$ unit/day to $10.6 \pm 1.00$ unit/day after 24 weeks $(P=0.04)$.

\subsection{DTSQ}

Although the scores of the DTSQ questionnaire did not significantly improve for "improvement of hypoglycemia” after switching $(P=0.901)$, all other 7 scores, namely "satisfaction", "improvement of hyperglycemia”, “convenience”, "versatility”, "recommendable to other patients”, "understanding”, and "treatment continuity" significantly improved $(P<0.001)$.

\section{Discussion}

The therapeutic concept of intensive insulin therapy is aimed to substitute the complex pattern of endogenous insulin secretion in patients with diabetes. The key features of a normal insulin profile include a sustained and relatively constant basal level of insulin secretion, along with a meal-stimulated peak (30 - 60 min) of insulin secretion that slowly decays over the subsequent 2 - $3 \mathrm{~h}$ [10]. The aim of subcutaneous injections of rapid-acting insulin before meals (bolus injection) is to mirror this meal-stimulated insulin secretion, whereas the aim of retarded insulin preparations is to substitute the basal level of insulin secretion. However, regarding bolus injections in particular, it was reported that rapid-acting insulin analogues such as insulin Lis or insulin Asp result in preprandial hypoglycemia or weight gain [7] [8], which could be attributable to the pharmacokinetic (PK) and pharmacodynamic (PD) properties of these two rapid-acting insulin analogues. Namely, these subcutaneously injected rapid-acting insulin analogues have slow onset of action compared with physiological endogenous insulin secretion (with a peak metabolic effect at approximately $1 \mathrm{~h}$ post-administration) and a prolonged duration of action beyond $3 \mathrm{~h}$ [11] [12], which inhibits achievement of good postprandial blood glucose control without development of preprandial hypoglycemia.

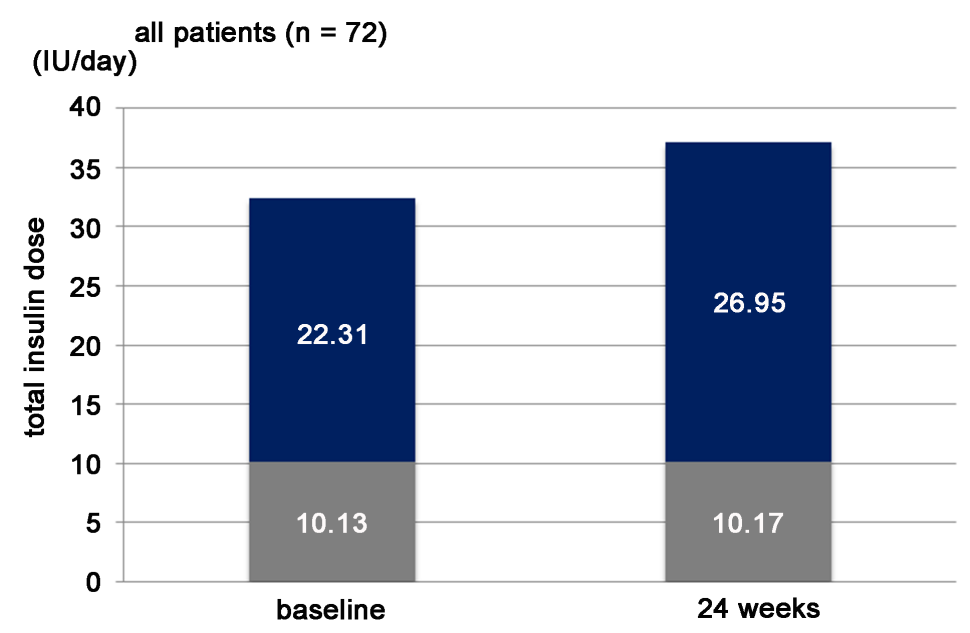

Figure 3. Changes in daily insulin dose from baseline after switching to insulin glulisine. Black and grey columns represent the mean total doses of bolus and basal insulin, respectively. 
Glu (B3-Lys-29B-Glu-insulin) is a rapid-acting insulin analogue developed to more closely resemble the physiological post-prandial insulin release and therefore, improve prandial glycemic control [13]. Glu has a unique zinc-free molecular structure that differs from other rapid-acting insulin analogues by the replacement of asparagine at position B3 by lysine, and of lysine at position B29 by glutamic acid of the human insulin molecule, and exists largely as a monomer in products, which are key features for rapid absorption from subcutaneous tissues [14] [15]. Glu is rapidly absorbed and immediately excreted in the bloodstream after subcutaneous injection, has a shorter time to peak metabolic effect (approximately 30 - 60 min after administration) and a reduced duration of action within $3 \mathrm{~h}$ (application document of Sanofi-Aventis: currently Sanofi). Therefore, Glu could ensure a good postprandial blood glucose control without development of preprandial hypoglycemia. Some previous reports described Glu to have a faster onset of action than Lis, independent of BMI and dose, in non-diabetic subjects [16] [17], and to achieve significant lower glucose level deviations than Lis in patients with type 2 diabetes [10].

In this study, we enrolled 72 outpatients with diabetes with inadequate glycemic control despite intensive insulin therapy with Gla plus Lis for $>24$ weeks. Treatment was switched from Lis to Glu by the addition of 1 unit of Glu at every meal that was increased in a stepwise manner thereafter to achieve a GA level $\leq 20 \%$. Six months after switching, both HbA1c and GA levels significantly lowered without any change in body weight. Furthermore, switching from Lis to Glu significantly improved patients' treatment satisfaction based on DTSQ, except for the "improvement of hypoglycemia", which did not show any significant changes despite the increase in bolus and basal insulin doses. We believe that the improved glycemic control after switching from Lis to Glu is mainly attributable to the reduced postprandial glucose deviations owing to the above-mentioned differences in the PK and PD properties between Lis and Glu. Our hypothesis is also partly supported by the non-significant reduction in the GA/HbA1c ratio, known to be a marker for postprandial glycemic excursion [18] [19]. The significant improvement in patients' satisfaction indicated by the DTSQ was probably associated with the improvement in blood glucose level control and the convenience of the simultaneous injection of Glu and Gla using the same injection devices $\left(\right.$ SoloSTAR ${ }^{\circledR}$ ).

\section{Study Limitations}

The first one is the one-arm, open-label, before-after study design and the limited number of subjects studied. Therefore, the possibility that the Hawthorn effect was partly associated with favorable effects after switching from Lis to Glu cannot be excluded. Furthermore, the 6-months study duration may be a second limitation because we are unable to sufficiently assess the effect of seasonal variation in glycemic control [20], despite the relatively long-term entry period of this study protocol ( 2 years). The third limitation is that the titration protocol used before and after the switching could bring bias results because of the subjective decision-making by the treating physician, despite the four treating physicians and target GA level that they aimed were all same before and after the switching. The fourth limitation is that change in the frequency of hypoglycemia was only evaluated using the score of "improvement of hypoglycemia" obtained by the DTSQ. Self-monitoring of blood glucose or continuous glucose monitoring CGM [21] data are required for a more accurate comparison.

\section{Conclusion}

Our present preliminary design of study suggests that switching bolus insulin from Lis to Glu by the addition of 1 unit of Glu per meal may be a useful treatment option for type 1 and type 2 diabetic patients with inadequate glycemic control receiving intensive insulin therapy with Lis and Gla, and can improve patients' treatment satisfaction without increasing the risk of hypoglycemia. However, to confirm these findings, a parallel-group comparison analysis in a larger population for a longer duration and using more detailed clinical parameters is warranted.

\section{Acknowledgements}

No funding or sponsorship was received for the medical writing assistance and article processing charges of this article. We thank current and former colleagues in the department of Internal Medicine at Fukui-ken Saiseikai Hospital. All named authors meet the ICMJE criteria for authorship for this manuscript, take responsibility for the integrity of the work as a whole, and have given final approval for the version to be published. 


\section{Conflict of Interest}

Y. Bando has received honoraria for consulting and lecturing from Sanofi-Aventis, Novartis and Eli Lilly. Other authors have no conflict of interest statement.

\section{Compliance with Ethics Guidelines}

The study was approved by the Ethics Committee of Fukui-ken Saiseikai Hospital.

All procedures followed were in accordance with the ethical standards of the responsible committee on human experimentation (institutional and national) and with the Helsinki Declaration of 1975, as revised in 2000 and 2008. Informed consent was obtained from all patients for being included in the study.

\section{References}

[1] Stamler, J., Vaccaro, O., Neaton, J.D. and Wentworth, D. (1993) Diabetes, Other Risk Factors, and 12-Yr Cardiovascular Mortality for Men Screened in the Multiple Risk Factor Intervention Trial. Diabetes Care, 16, 434-444.

http://dx.doi.org/10.2337/diacare.16.2.434

[2] Haffner, S.M., Lehto, S., Rönnemaa, T., Pyörälä, K. and Laakso, M. (1998) Mortality from Coronary Heart Disease in Subjects with Type 2 Diabetes and in Nondiabetic Subjects with and without Prior Myocardial Infarction. New England Journal of Medicine, 339, 229-234. http://dx.doi.org/10.1056/NEJM199807233390404

[3] The DECODE Study Group (1999) Glucose Tolerance and Mortality: Comparison of WHO and American Diabetes Association Diagnostic Criteria. The DECODE Study Group. European Diabetes Epidemiology Group. Diabetes Epidemiology: Collaborative Analysis of Diagnostic Criteria in Europe. Lancet, 354, 617-621.

[4] Tominaga, M., Eguchi, H., Manaka, H., Igarashi, K., Kato, T. and Sekikawa, A. (1999) Impaired Glucose Tolerance Is a Risk Factor for Cardiovascular Disease, But Not Impaired Fasting Glucose. The Funagata Diabetes Study. Diabetes Care, 22, 920-924. http://dx.doi.org/10.2337/diacare.22.6.920

[5] Donahue, R.P., Abbott, R.D., Reed, D.M. and Yano, K. (1987) Postchallenge Glucose Concentration and Coronary Heart Disease in Men of Japanese Ancestry. Honolulu Heart Program. Diabetes, 36, 689-692. http://dx.doi.org/10.2337/diab.36.6.689

[6] 2011 Guideline for Management of PostMeal Glucose in Diabetes. www.idf.org

[7] Holman, R.R., Farmer, A.J., Davies, M.J., et al. (2009) Three-Year Efficacy of Complex Insulin Regimens in Type 2 Diabetes. The New England Journal of Medicine, 361, 1736-1747. http://dx.doi.org/10.1056/NEJMoa0905479

[8] Daikubara, H., Kikuti, T. and Ishida, T. (2011) Shinyaku to Rinsho, 60, 758-765 (in Japanese).

[9] Pouwer, F., Snoek, F.J., van der Ploeg, H.M., Heine, R.J. and Brand, A.N. (1998) A Comparison of the Standard and the Computerized Versions of the Well-Being Questionnaire (WBQ) and the Diabetes Treatment Satisfaction Questionnaire (DTSQ). Quality of Life Research, 7, 33-38. http://dx.doi.org/10.1023/A:1008832821181

[10] Luzio, S., Peter, R., Dunseath, G.J., Mustafa, L. and Owens, D.R. (2008) A Comparison of Preprandial Insulin Glulisine versus Insulin Lispro in People with Type 2 Diabetes over a 12-h Period. Diabetes Research and Clinical Practice, 79, 268-275. http://dx.doi.org/10.1016/j.diabres.2007.11.013

[11] Plank, J., Wutte, A., Brunner, G., Siebenhofer, A., Semlitsch, B., Sommer, R., et al. (2002) A Direct Comparison of Insulin Aspart and Insulin Lispro in Patients with Type 1 Diabetes. Diabetes Care, 25, 2053-2057. http://dx.doi.org/10.2337/diacare.25.11.2053

[12] Homko, C., Deluzio, A., Jimenez, C., Kolaczynski, J.W. and Boden, G. (2003) Comparison of Insulin Aspart and Lispro: Pharmacokinetic and Metabolic Effects. Diabetes Care, 26, 2027-2031. http://dx.doi.org/10.2337/diacare.26.7.2027

[13] Garg, S.K., Rosenstock, J. and Ways, K. (2005) Optimized Basal-Bolus Insulin Regimens in Type 1 Diabetes: Insulin Glulisine versus Regular Human Insulin in Combination with Basal Insulin Glargine. Endocrine Practice, 11, 11-17. http://dx.doi.org/10.4158/EP.11.1.11

[14] Becker, R.H. and Frick, A.D. (2008) Clinical Pharmacokinetics and Pharmacodynamics of Insulin Glulisine. Clinical Pharmacokinetics, 47, 7-20. http://dx.doi.org/10.2165/00003088-200847010-00002

[15] Garg, S.K., Ellis, S.L. and Ulrich, H. (2005) Insulin Glulisine: A New Rapid-Acting Insulin Analogue for the Treatment of Diabetes. Expert Opinion on Pharmacotherapy, 6, 643-651. http://dx.doi.org/10.1517/14656566.6.4.643

[16] Becker, R.H., Frick, A.D., Burger, F., Potgieter, J.H. and Scholtz, H. (2005) Insulin Glulisine, a New Rapid-Acting Insulin Analogue, Displays a Rapid Time-Action Profile in Obese Non-Diabetic Subjects. Experimental and Clinical Endocrinology \& Diabetes, 113, 435-443. http://dx.doi.org/10.1055/s-2005-865806 
[17] Heise, T., Nosek, L., Spitzer, H., Heinemann, L., Niemöller, E., Frick, A.D. and Becker, R.H.A. (2007) Insulin Glulisine: A Faster Onset of Action Compared with Insulin Lispro. Diabetes, Obesity and Metabolism, 9, 746-753. http://dx.doi.org/10.1111/j.1463-1326.2007.00746.x

[18] Sakura, N., Omura, M., Oda, E. and Saito, T. (2011) Converse Contributions of Fasting and Postprandial Glucose to $\mathrm{HbA}_{1 \mathrm{c}}$ and Glycated Albumin. Diabetology International, 2, 162-171. http://dx.doi.org/10.1007/s13340-011-0036-9

[19] Imai, T., Oikawa, Y. and Shimada, A. (2007) Improved Monitoring of the Hyperglycemic State in Type 1 Diabetes Patients by Use of the Glycoalbumin/HbA1c Ratio. The Review of Diabetic Studies, 4, 44-48. http://dx.doi.org/10.1900/RDS.2007.4.44

[20] Higgins, T., Saw, S., Sikaris, K., Wiley, C.L., Cembrowski, G.C., Lyon, A.W., et al. (2009) Seasonal Variation in Hemoglobin A1c: Is It the Same in both Hemispheres? Journal of Diabetes Science and Technology, 3, 668-671. http://dx.doi.org/10.1177/193229680900300408

[21] DeSalvo, D. and Buckingham, B. (2013) Continuous Glucose Monitoring: Current Use and Future Directions. Current Diabetes Reports, 13, 657-662. 
Scientific Research Publishing (SCIRP) is one of the largest Open Access journal publishers. It is currently publishing more than 200 open access, online, peer-reviewed journals covering a wide range of academic disciplines. SCIRP serves the worldwide academic communities and contributes to the progress and application of science with its publication.

Other selected journals from SCIRP are listed as below. Submit your manuscript to us via either submit@scirp.org or Online Submission Portal.
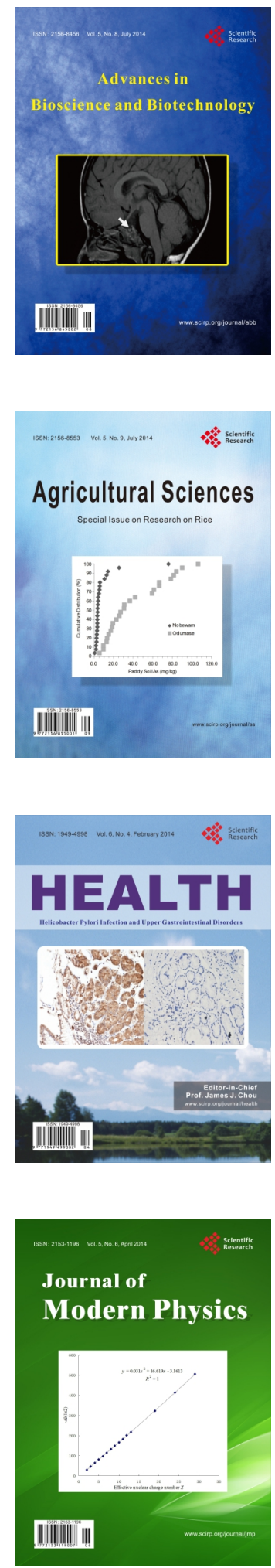
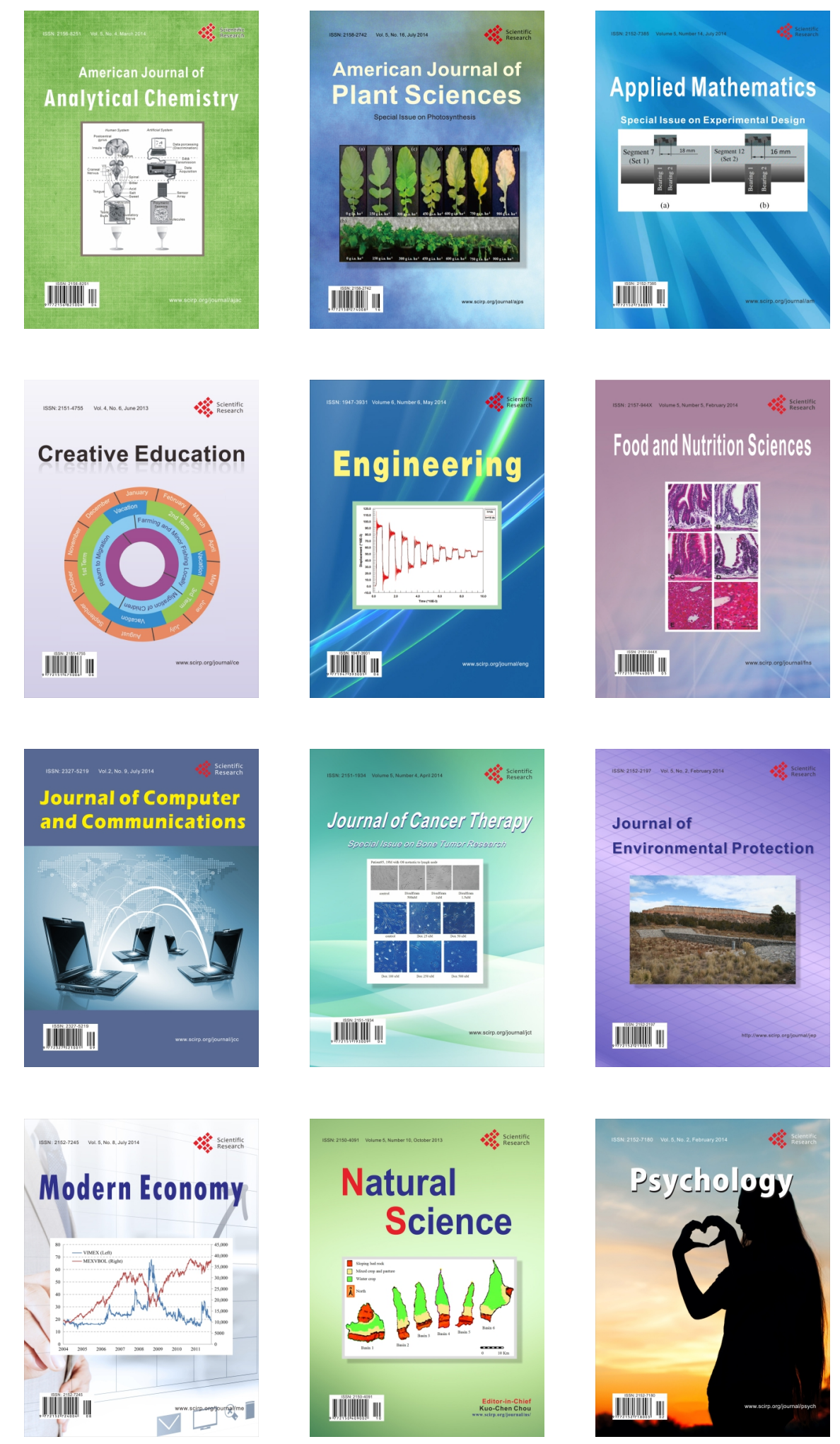